\title{
Susceptibility of rhesus monkeys (Macaca mulatta) to the Ponzo illusion
}

\author{
KATHRYN A. L. BAYNE and ROGER T. DAVIS \\ Washington State University, Pullman, Washington
}

\begin{abstract}
Four rhesus monkeys (Macaca mulatta) showed susceptibility to the Ponzo illusion. This is a unique result, since the Ponzo employs extraneous inducing components and monkeys must be trained to ignore these components.
\end{abstract}

There are quite a few two-dimensional geometric drawings that are perceived as illusions by human beings. On the other hand, relatively few of these drawings have been demonstrated to be unambiguously illusory in nonhuman subjects. The capability of subjects without language to perceive illusions is still undetermined. Several claims of illusion in animals are questionable because of the availability of proprioceptive as well as visual cues. Some supposedly illusory figures produce ambiguous results with animals because of the difficulty of instructing nonverbal subjects which part of a figure should be attended such as the straight line and which part should be ignored, such as the arrows in the Müller-Lyer figure.

Some time ago, Dominguez (1954) demonstrated that monkeys are more likely to see rectangles as being taller than squares (breadth-of-rectangles illusion). This was corroborated later by Harris (1968) using very different methods from those used by Dominguez. One difference between this illusion and many others is that the illusion is independent of parts of the figure that must be ignored. Harris recognized that the breadth-of-rectangles illusion, for example, can be an artifact of area, but with area controlled, the illusion persists with monkeys, and in fact there is a functional relationship between the width of the figures and the degree of the illusion (Davis, 1974). Recently, Benhar and Samuel (1982) demonstrated the susceptibility of two baboons to the Zöllner illusion. Unlike the breadth-of-rectangles figure, the Zöllner illusion depends on the presence of inducing parts of the figure. They employed an outside-oddity paradigm, which allows an experimenter to ask questions of an animal about differentness.

Assuming for a moment that some drawings are perceived as illusory by some animals, we should next ask if illusions in general are compelling to animals if appropriate experimental methods are used.

The present study was an attempt to extend the list of figures that are illusory to nonhumans and to see if

The authors' mailing address is: Department of Psychology, Washington State University, Pullman, Washington 99163. this is possible with inducing components that change the overall shape of the discriminated figure.

\section{METHOD}

\section{Subjects}

Two male and two female rhesus monkeys (Macaca mulatta) were tested for susceptibility to the Ponzo illusion. The subjects had been laboratory raised. One subject of each sex was 4 years old, and the other subject of each sex was 8 years old.

\section{Procedure}

The subjects were trained 25 trials/day, 7 days/week, to discriminate between Masonite blocks shaped as rectangles and trapezoids. The rectangular stimuli measured $8.0 \times 6.0 \mathrm{~cm}$. The trapezoidal stimuli were of three sizes: (1) $6.0 \times 8.0 \times 10.0 \mathrm{~cm}$; (2) $6.0 \times 8.0 \times 12.0 \mathrm{~cm}$; and (3) $6.0 \times 10.0 \times 12.0 \mathrm{~cm}$. The blocks were covered with white paper bordered heavily in black. All stimuli were presented vertically, with each stimulus being hung by transparent nylon thread from hooks separated by a distance of $19.5 \mathrm{~cm}$. The background for these stimuli was a plywood board painted gray and measuring $54.0 \times 69.5 \mathrm{~cm}$. A raisin reward was concealed behind the stimulus considered appropriate for each subject (i.e., half of the subjects were rewarded for making a left-hand response when two rectangles were present and a right-hand response when two trapezoids were displayed; the other subjects were rewarded to these stimuli on the opposite sides). During each experimental session, each animal was temporarily confined in a carrying cage that had a solid floor and ceiling, two grid side walls, one end with a Lucite door that had two holes large enough for the animal to reach through (thus eliminating any linear cues that might result from reaching through bars), and the other end of solid steel. The carrying cage was positioned such that stimuli were within arm's length of the animal. A guillotine-type door was lowered at the end of each trial to prevent the animal from watching the experimenter change the stimuli.

The animals were first trained to differentiate between the rectangular stimuli and the largest pair of trapezoidal stimuli in a successive-discrimination paradigm. The subjects were rewarded for going to the right for one pair of stimuli and to the left for the second pair of stimuli. The presentation of the stimuli was randomized by following the Gellerman series.

The criterion for successful discrimination was set at $80 \%$ for 3 consecutive days or $88 \%$ for 2 consecutive days. Once the subject had reached the criterion level for discriminating the rectangular stimuli from the largest trapezoidal stimuli, the middlesized trapezoids were substituted into the discrimination task. When criterion levels were once again reached for this discrimination, the smallest trapezoids were introduced. The purpose of using different-sized trapezoids was to obtain a generalized response to figures of this shape. When a criterion of success for 
the last task had been reached, the subject was presented with white test stimuli constructed of cardboard, upon which were drawn rectangles or trapezoids of the same size as the smallest training stimuli, embedded in an array of converging black lines (see Figure 1). The test stimuli measured $19.5 \times 17.5 \mathrm{~cm}$. They were presented every third trial, of 27 total trials each day, with each presentation being preceded and followed by one trial of rectangular or trapezoidal blocks (also randomized by following the Gellerman series). A double-reward system was utilized during presentation of the test stimuli so that any response resulted in reward. After 25 responses to the Ponzo figure (i.e., the rectangular stimulus embedded in the array of lines), the training was reversed. Thus, an animal trained to go left for rectangles

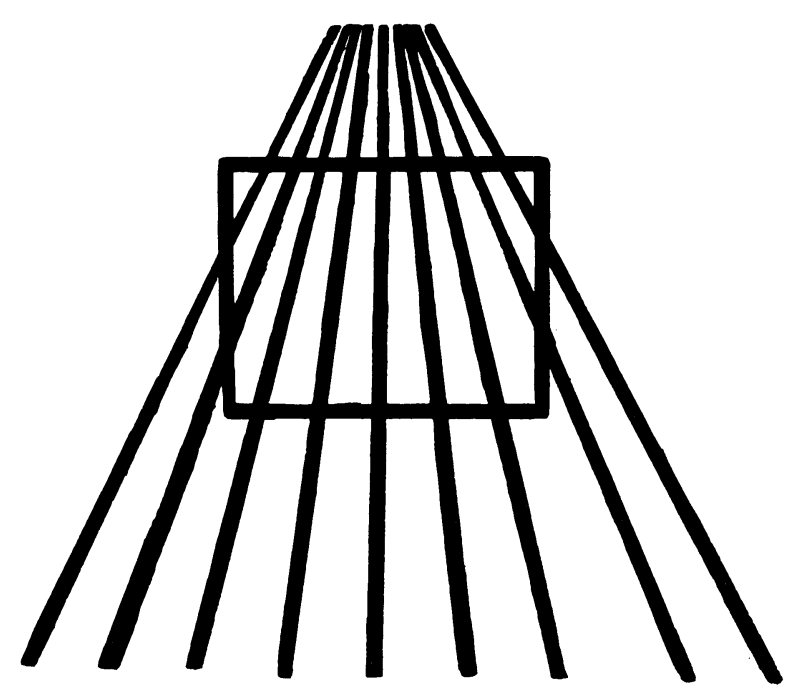

A. Rectangle

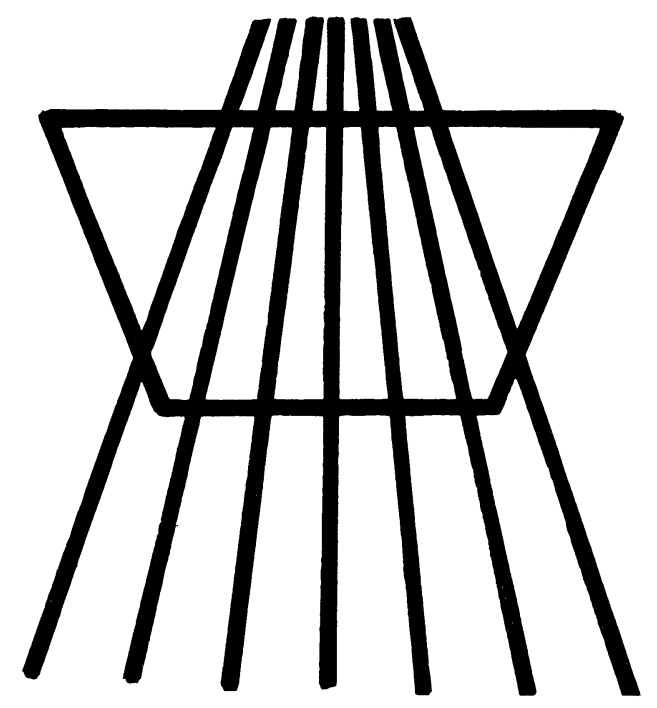

\section{B. Trapezoid}

Figure 1. Ponzo figure. (A) Rectangle embedded in the array: (B) trapezoid embedded in the array.
Table 1

Number of Times (of 25) Subject Was Affected by the Illusion

\begin{tabular}{lccccc}
\hline Males & Females & $\begin{array}{c}\text { Original } \\
\text { Training }\end{array}$ & $\mathrm{p}<$ & Reversal & $\mathrm{p}<$ \\
\hline 124 & & 23 & .001 & 25 & .001 \\
NIH 1 & & 25 & .001 & 23 & .001 \\
& 110 & 17 & .054 & 22 & .001 \\
& 123 & 16 & .115 & 22 & .001 \\
\hline
\end{tabular}

Note-The $p$ values indicate the probability that the results were obtained by chance; the binomial test was used.

and right for trapezoids would next go right for rectangles and left for trapezoids. This eliminated the possibility of the position habit's affecting the results. The same criterion of success was required before the test stimuli were presented during the reversal trials.

\section{RESULTS}

All the animals reached the criterion for success in discriminating the rectangular stimuli from the smallest trapezoidal stimuli in fewer than 900 trials. Criterion levels were reached even faster under the reversal condition (300 or fewer trials).

An animal was considered to be susceptible to the Ponzo illusion if it responded to the side that was appropriate for a trapezoidal figure. When presented with the Ponzo illusion during the first determination, the males responded to it as if it were a trapezoid embedded in an array of lines $92 \%-100 \%$ of the time. The females, on the other hand, responded to the illusion as if it were a trapezoid $64 \%-68 \%$ of the time. Under the reversal condition, the difference between the males and females was not as dramatic. The males still responded to the illusion as if it were a trapezoid $92 \%-100 \%$ of the time, and the females were affected by the illusion $88 \%$ of the time.

A binomial test showed that the animals were affected by the illusion significantly more often than they were not. As can be seen from Table 1, six of eight cells contain figures that occur with a significance of $\mathrm{p}<.001$, thus indicating that the subjects were susceptible to this particular illusion and were able to reverse their position responses.

\section{DISCUSSION}

It can be concluded from the results of this study that the illusory properties of the Ponzo figure may be manifested in monkeys. This finding tends to indicate that inducing parts of the figure can have an illusory effect on nonhuman beings and is in accord with Benhar and Samuel's (1982) similar findings. It does not agree with Gregory's (1972) hypothesis that familiarity with converging lines is necessary in order to see the illusion, since monkeys are not familiar with railroad tracks and the like, but does continue to support the hypothesis that there are many similarities between the visual abilities of monkeys and human beings (DeValois \& Jacobs, 1971).

The difference in susceptibility to the illusion between the sexes was not significant. The females did not appear to be as susceptible as the males to the illusion during the first determina- 
tion, but this difference was not as evident, however, during the second determination. This sex difference was not significant, but its direction was opposite to what had been found with human beings. This possible contradictory aspect of the findings needs further investigation.

The key factor in the Ponzo illusion seems to be the conflicting information in the figure concerning size constancy and distance (Leibowitz, 1965). The subjects in this study were able to make a choice of one geometric figure or the other. Davis (1974), however, found that monkeys in the Ames distorted room could not resolve the conflict of these two cues. He concluded that conflicting information was more confusing to monkeys than to human beings. On the basis of the results of this study, this question also needs to be investigated further.

Since the question of whether visual information processing in monkeys is similar to that in human beings was not completely answered, a desirable extension of the present study would be to abstract various aspects of the illusory figures for tests to determine which parts of the figures are appropriate stimuli to produce choice. It is entirely possible that the angle between the outermost lines of the array and the underlying figure is the most important cue in determining the response. Maybe the fact that the trapezoid has an angled side results in the choice of the display with the lines superimposed over the rectangle, since the superimposed lines slant, although in the opposite direction from the sides of the trapezoid in the training figure. Possibly, the subjects have obtained a generalized concept of slope and interpret the diverging superimposed line as a slope.

The present investigators were planning further research into the potency of various aspects of the figures as illusory stimuli, but that work was made impossible by changes in laboratory conditions. Such research would aid in identifying optical variables in illusions. It might also clarify the possibility that human beings might be attending to particular aspects of an illusory figure rather than to the whole figure. If this is the case, the subjects may not be "susceptible" to illusions in the usual sense, but may be making choices based on geometric cues instead.

\section{REFERENCES}

Benhar, E., \& Samuel, D. Visual illusions in the baboon (Papio anubis). Animal Learning \& Behavior, 1982, 10, 115-118.

DAvis, R. T. Monkeys as perceivers. In L. A. Rosenblum (Ed.), Primate behavior: Developments in field and laboratory research (Vol. 3). New York: Academic Press, 1974.

DeVAlois, R. L., \& JAcoBs, G. H. Vision. In A. M. Schrier \& F. Stollnitz (Eds.), Behavior of nonhuman primates: Modern research trends (Vol. 3). New York: Academic Press, 1971.

Dominguez, K. E. A study of visual illusions in the monkey. Journal of Genetic Psychology, 1954, 85, 105-127.

GREGORY, R. L. Eye and brain. London: World University Library, 1972.

Harris, A. V. Perception of the horizontal-vertical illusion in stumptail monkeys. Radford Review, 1968, 22, 61-72.

Leibowitz, H. W. Visual perception. New York: Macmillan, 1965.

(Manuscript received for publication August 18, 1983.) 